Troponinerhöhungen bei allen kritisch Kranken möglich

Neben dem Typ-1- und Typ-2-Infarkt, denen immer eine ischämische Myokardschädigung zugrunde liegt, gibt es nicht infarktbedingte Troponinerhöhungen. „Von allen Patienten einer CPU haben nur 20 Prozent einen erhöhten Troponinwert und bei 69 Prozent dieser Patienten liegt kein ACS vor", erklärte Katus. Bei nahezu allen akuten kritischen Erkrankungen unabhängig davon, ob sie neurologischer, infektiöser, pulmonaler, onkologischer oder gastroenterologischer Art seien, könne das Troponin erhöht sein, ohne dass ein ACS vorliege.

Der genaue Mechanismus der Troponinfreisetzung ist in diesen Situationen unklar. Man muss annehmen, dass bei solchen Erkrankungen in irgendeiner Form das Herz mitbeteiligt ist. Doch Patienten mit schweren akuten Erkrankun- gen und nicht infarktbedingtem Troponinanstieg haben unabhängig von der Grundkrankheit ein erhöhtes Sterberisiko und sollten daher kardiologisch überwacht und betreut werden.

„Dies zeigt, dass man mit dem hs-Troponin mehr entdecken kann, als man oberflächlich sieht“, schloss Katus.

Dr. med. Peter Stiefelhagen

\title{
TAVI-Nachsorge: Worauf man achten sollte
}

\section{TAVI-Patienten erfordern eine lebenslange Nachsorge. Dabei geht es einmal um die Beurteilung der Klappenfunktion, zum anderen um das Management der antithrombotischen Begleittherapie und Endokarditisprophylaxe.}

$\mathrm{D}$ ie Zahl der Patienten mit Transkatheter-Aortenklappenimplantation (TAVI) wächst ständig; für inoperable Patienten ist die TAVI alternativlos, für Hochrisikopatienten stellt sie die bessere Option dar. Doch auch TAVI-Patienten erfordern ebenso wie Patienten nach chirurgischem Klappenersatz eine lebenslange Nachbetreuung, wobei spezifische Aspekte zu berücksichtigen sind.

Die Leitlinie empfiehlt regelmäßige Kontrollen nach drei und sechs Monate und dann alle zwölf Monate. Diese umfassen eine sorgfältige klinische Untersuchung, wobei besonders nach Dyspnoe und reduzierter Belastbarkeit gefragt werden sollte. Unverzichtbar sind ein EKG und eine transthorakale Echokardiografie. Im Echo sollte man auf einen Anstieg des transvalvulären Druckgradienten, Segelverdickungen und -beweglichkeit achten. Je nach Klappentyp kommt es bei bis zu $40 \%$ der Patienten sowohl zu einer Störung der Klappenöffnung mit deutlichem transvalvulären Druckanstieg als auch zu einer Schlussundichtigkeit. Als Ursache werden kleine thrombotische Auflagerungen vermutet. „Dafür spricht, dass sich diese Prothesendysfunktion nach Einleitung einer oralen Antikoagulation mit Marcumar fast immer vollständig zurückbildet“, sagte Prof. Jochen Wöhrle, Universitätsklinikum Ulm. Bisher wisse man aber nicht, ob man die OAK dauerhaft fortsetzen müsse. Auch auf das Vorlie- gen eines paravalvulären Lecks sollte man achten. Dieses ist heute bei Klappen der 3. Generation mit einer Inzidenz von circa $2 \%$ sehr selten. Wenn nötig kann die Leckage mit einem Okkluder verschlossen werden.

\section{LSB hat prognostische Bedeutung}

Tritt beim interventionellen Klappenersatz ein höhergradiger AV-Block auf, so ist ein Schrittmacher nötig. Ein Linksschenkelblock (LSB) allein stellt aber noch keine Indikation für einen Schrittmacher dar, sondern erst, wenn ein AVBlock I0 hinzukommt. Ein LSB hat aber eine gewisse prognostische Bedeutung; die durch den Klappenersatz bedingte Besserung der eingeschränkten linksventrikulären Funktion ist im Falle eines LSB beeinträchtigt, aber nur, wenn die Ejektionsfraktion (EF) primär $<35 \%$ betrug. Der Schrittmacher selbst hat bei TAVI-Patienten aber keinerlei Einfluss auf die Prognose.

\section{Welche Begleittherapie?}

Evidenzbasierte Leitlinienempfehlungen zur antithrombotischen Therapie bei TAVI gibt es bisher nicht. Somit ist die Vorgehensweise empirisch begründet und nicht einheitlich. Bei der antithrombotischen Therapie geht es darum, das periprozedural erhöhte zerebroembolische Risiko zu minimieren. Die Inzidenz klinisch manifestierter Hirnembolien liegt heute dank moderner Em-
bolie-Protektionssysteme bei unter $2 \%$. „Doch MRT-Untersuchungen haben gezeigt, dass in etwa 80 bis 100 Prozent der Fälle stumme ischämische Hirnläsionen auftreten“, sagte Wöhrle. Generell empfohlen wird eine ASS-Therapie und zwar lebenslang, wenn keine OAK erfolgt. Bei der CorValve-Prothese sollte man in den ersten vier Wochen zusätzlich Clopidogrel geben. Bei Patienten mit OAK sollte hier nur in den ersten vier Wochen zusätzlich Clopidogrel verabreicht werden. Bei leichteren Blutungen sollte man einen der beiden Plättchenhemmer oder bei OAK-Patienten Clopidogrel absetzen. „Ob die duale Strategie wirklich effektiver ist als eine ASS-Monotherapie oder ob dadurch nur das Blutungsrisiko erhöht wird, weiß niemand“, so Wöhrle.

Bei einer SAPIEN-Prothese wird von Anfang an nur eine ASS-Monotherapie empfohlen, bei einer OAK sollte sogar auf die zusätzliche ASS-Gabe völlig verzichtet werden. Gefürchtet sind schwere Blutungskomplikationen, die eine Unterbrechung der antithrombotischen Therapie nötig machen können, was wiederum zu vermehrten ischämischen Insulten führt. Aktuell laufen Studien, die die noch offenen Fragen beantworten sollen: ob eine duale Plättchenhemmung effektiver und sicher ist bzw. ob ein $\mathrm{Pa}$ tient mit einem NOAK von der zusätzlichen ASS-Gabe profitiert. „Vielleicht wäre es auch sinnvoll, alle TAVI-Patienten drei Monate zu antikoagulieren wie bei einer Bioprothese“, sagte Wöhrle.

TAVI-Patienten sind auch Hochrisikopatienten bzgl. einer Endokarditis. Sie erfordern daher eine Antibiotika-Prophylaxe, aber nur bei gingivalen Eingriffen, einer transbronchialen Biopsie oder bei urologischen Eingriffen. 